\section{(6) OPEN ACCESS}

For numbered affiliations see end of article.

\section{Correspondence to}

Dr N Pathmanathan, Westmead Breast Cancer Institute, Westmead Hospital, Westmead, NSW 2145, Australia;

Nirmala.Pathmanathan@bci. org.au

Received 4 June 2013 Revised 27 August 2013 Accepted 31 August 2013 Published Online First 8 January 2014

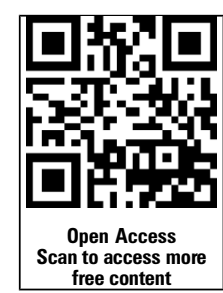

To cite: Pathmanathan N Balleine RL, Jayasinghe UW, et al. I Clin Pathol 2014;67:222-228.

\title{
The prognostic value of Ki67 in systemically untreated patients with node-negative breast cancer
}

\author{
Nirmala Pathmanathan, ${ }^{1,2,3}$ Rosemary L Balleine, ${ }^{3,4,5,6}$ Upali W Jayasinghe, ${ }^{1,7}$ \\ Kellie L Bilinski, ${ }^{1}$ Pamela J Provan, ${ }^{4,5,6}$ Karen Byth, ${ }^{3}$ A Michael Bilous, ${ }^{1,2,8}$ \\ Elizabeth L Salisbury, ${ }^{1,2,9}$ John Boyages ${ }^{1,10}$
}

\section{ABSTRACT}

Aim To evaluate the utility of Ki67 as a prognostic marker in a series of patients with node-negative breast cancer untreated with adjuvant systemic therapy. Methods The cohort consisted of 203 cases treated with breast conserving surgery and radiation only; median follow-up was 183 months (range 156-277 months). An immunohistochemical panel of oestrogen receptor (ER), progesterone receptor (PR), cytokeratin (CK)5/6 and Ki67 and human epidermal growth factor 2 in situ hybridization (HER2-ISH) was performed on the tumour samples. Ki67 scores were evaluable in 193/203 patients (95.1\%). The primary outcome was breast cancer specific survival (BCSS). Results Of the cohort, 29 (14.2\%) died of breast cancer. A cut off of $10 \%$ separated tumours into a 'Ki67-low' ( $n=70)$ or 'Ki67-high' group ( $n=123)$. The breast cancer specific survival was $97.1 \%$ and $77.6 \%$ for Ki67-low and Ki67-high groups, respectively. Univariate analysis showed that in this lymph node-negative cohort, the predictors for BCSS were tumour size, Ki67, LVI, age and histological grade 3. Multivariable analysis showed that Ki67 index and lymphovascular space invasion were independent predictors of breast cancer death. To examine the utility of Ki67 in assignment of immunohistochemically molecular subtypes, cases were assigned into Luminal A (ER-positive, HER2-negative, Ki67 $\leq 14 \%$ ), Luminal B (ER-positive, HER2-negative, Ki67 >14\%) and triple negative (ER/PR-negative and HER2-negative, any Ki67). The 15 -year breast cancer specific survival was $91.7 \%$, $79.4 \%$ and $75.8 \%$, respectively.

Conclusions A statistically significant difference in breast cancer specific survival is seen in groups defined using Ki67 and receptor status, whereas histological grading was not a significant predictor of survival. Ki67 immunostaining provides prognostic information beyond traditionally assessed clinicopathological variables.

\section{INTRODUCTION}

Prognostication and treatment selection for patients with breast cancer is reliant on the assessment of clinical and biological characteristics of breast tumours. Gene expression profiling studies ${ }^{1} 2$ have served to further emphasise the underlying genetic heterogeneity of breast cancers and this is reflected clinically in terms of prognosis and treatment response. The classification of breast cancers into clinically meaningful subgroups on the basis of gene expression profiles is relevant to contemporary oncology practice. There is an immediate need to further refine the prognostic and predictive assessment of breast cancer in order to identify optimal, tailored therapeutic regimens for individual patients and identify those patients in which adjuvant therapy can be avoided. Consequently, there is interest in application of these molecular techniques to routine practice, as evidenced by the publication of several studies defining various molecular signatures as well as the increasing use of commercially available multigene assays. ${ }^{3}$ Significantly, there is little overlap in terms of individual genes in most of these assays; notably, however, proliferation related genes appear to be a common discriminatory component across all array platforms. ${ }^{3}{ }^{4}$ Indeed, the most widely used assay, Oncotype DX (Genomic Health, Redwood City, California, USA), calculates a recurrence score derived from the differential expression of 16 discriminatory genes, with MK167 (encoding Ki67) being the most heavily weighted component of the formula. ${ }^{4}$

In general, time dependant prognostic factors such as tumour size and lymph node metastasis may not declare their associated risk at the time of diagnosis. This is especially relevant in early breast cancer and in a setting of population-based mammographic screening. In this regard, the biological/ molecular characteristics of breast cancer assume even greater significance, however, given the prohibitive costs and technical difficulty associated with molecular profiling studies, there is renewed interest in more detailed evaluation of histopathological features including immunohistochemical markers to optimise risk evaluation.

Adjuvant therapy is a confounding factor to understanding the true biological potential of breast cancer. For example, mortality patterns in women with oestrogen receptor (ER)-positive cancers untreated with adjuvant hormone therapy have been shown to be similar to those in women with ER-negative cancers. ${ }^{5}$ Therefore, there is an advantage in evaluating cancers from patients previously untreated by adjuvant systemic therapy. The aim of this study was to evaluate Ki67 immunohistochemistry as a prognostic marker in isolation and combined with other routinely used biomarkers in a cohort of patients with node-negative breast cancer untreated with adjuvant systemic therapy.

\section{PATIENTS AND METHODS \\ Cohort characteristics}

Individuals included in this study were from a previously described cohort comprising a consecutive series of 421 patients with pathologically lymph 
node-negative breast cancer who were treated with breastconserving surgery and postoperative radiotherapy at Westmead Hospital, Westmead, Australia between November 1979 and December $1994 .{ }^{6}$ All patients underwent axillary dissection and only patients with a minimum of five dissected lymph nodes were included. ${ }^{7}$ Overall, 203 patients were included in this study on the basis of availability of a paraffin tumour block. The study was conducted with institutional Human Research Ethics Committee approval.

Information on date of death with cancer as the cause of death was obtained from the New South Wales Central Cancer Registry via data linkage performed by the Centre for Health Records and Data Linkage (CHeReL, http://www.cherel.org.au). The creation of the dataset by data linkage followed several processes. Firstly, data from the Central Cancer Registry and Admitted Patient Data Collection was linked by CHeReL using probabilistic methods and Choicemaker software. Variables used in this probabilistic linkage process include name, address, date of birth, sex, country of birth, hospital code and medical record number. Groups with intermediate probabilities (in the range $0.25-0.75$ ) were subject to clerical review. The estimated false positive and false negative rate for this linkage was around $0.5 \%$. The resulting dataset was then linked to the Breast Conservation database (who were treated with breast-conserving surgery and postoperative radiotherapy). The first linkage pass was deterministic and used surname, date of birth, sex and the first initial of given name. Unlinked records from the Breast Conservation Database were then subject to a full probabilistic pass. Thresholds were set so that false positive rates did not exceed $0.5 \%$. Where full identifiers are available, the false negative rate is estimated at $0.5 \%$.

In the absence of death, patients were assumed to be alive as of 31 December 2007, the latest available data for this study. The follow-up period was defined as time from surgery to last date of follow-up for censored cases, or death for complete observation. The median follow-up period for patients was 183 months (range, 156-277 months).

\section{Histopathological review and tissue microarray construction}

Details of centralised pathology review have been previously described. ${ }^{6}$ Documented pathological features included histological type ${ }^{8}$ histological grade, ${ }^{9}{ }^{10}$ and presence of lymphovascular invasion by breast cancer (LVI). Viable tumour samples from different areas of paraffin blocks were selected for inclusion in tissue microarrays (TMA) with three $0.6 \mathrm{~mm}$ cores sampled from each case using a manual arrayer (Beecher Instruments, Maryland, USA). Slides, prepared from $4 \mu \mathrm{m}$ thick sections, were stained with haematoxylin and eosin (H\&E) together with an immunohistochemical panel and human epidermal growth factor 2 (HER2) silver in situ hybridisation (ISH; see below).

\section{Immunohistochemical staining}

Ki67 staining was performed on the Ventana Benchmark autostainer (following mild CC1 pretreatment) using the primary antibody clone SP6 (Thermo Fisher Scientific, Fremont, California, USA), at 1:200 dilution with detection using UltraView (Ventana Medical Systems Tucson, Arizona) detection kit. For Ki67 assessment, the slides were scanned at $\times 400$ magnification using a ScanScope CS (Aperio Technologies, Vista, California, USA), and digital images were visualised using ImageScope V.6 (Aperio). Individual positive and negative cells were counted using the manual tag function of Image Pro Plus V.5 (Media Cybernetics, Bethesda, Maryland, USA). All cores were examined and those cores with the greatest number of positive staining tumour cell nuclei were selected for counting, with additional cores counted where less than 1000 cells were present in a single core. All tumour cell nuclei with homogenous/granular or speckled staining or nucleolar staining were regarded as positive, regardless of staining intensity. ${ }^{11}$ Overall, a median of 1556 tumour cells per case (range 79-12 322), were counted. A total of 10 cases were not assessable for Ki67 due to inadequate tumour sample or unsatisfactory staining. The percentage of total cells that stained positive for Ki67 is reported as the Ki67 index.

Mouse monoclonal antibodies were used for detection of ER (clone 6F11, prediluted, Ventana Medical Systems) and progesterone receptor (PR) (clone 16, prediluted, Ventana Medical Systems), following pretreatment with mild CC1. Detection was performed using UltraView DAB kit (Ventana Medical Systems). Positive nuclear staining of $\geq 1 \%$ of tumour cells of any intensity was regarded as positive.

Cytokeratin (CK) $5 / 6$ staining was assessed as a surrogate marker of basal phenotype breast cancers, ${ }^{12}$ CK5/6 (Cellmarque, Rocklin, California, USA) at 1:100 dilution following pretreatment with mild CC1. Detection was with UltraView detection kit (Ventana Medical Systems). Staining was assessed as positive if $\geq 1 \%$ of cells showed positive cytoplasmic staining of any intensity.

A positive-control section was included in each staining run.

\section{HER2 amplification testing}

HER2 amplification was assessed using the single probe INFORM HER2 DNA kit according to manufacturer's instructions. The probe was labelled with dinitrophenol (DNP) used with the Ventana UltraView SISH Detection Kit on the Ventana BenchMark XT automated slide stainer. The repeat-sequencedepleted HER2 DNA probe was denatured at $95^{\circ} \mathrm{C}$ for $12 \mathrm{~min}$ followed by hybridisation at $52^{\circ} \mathrm{C}$ for $2 \mathrm{~h}$. The HER2 signal was visualised using the rabbit anti-DNP primary antibody and the UltraView SISH Detection Kit. Scoring of HER2 was according to criteria detailed in published guidelines. ${ }^{13}{ }^{14}$ Cases with a mean of $<4$ gene copies were designated as negative (nonamplified), cases with 4-6 copies were designated as equivocal, and cases with a mean copy number $\geq 6$ were designated as positive (amplified). In three cases, no result was obtained for HER2 status due to suboptimal staining or inadequate tumour sample.

\section{Statistical analyses}

Statistical analyses were performed using SPSS statistical software (PASW V.18; SPSS, Chicago, Illinois, USA) and SAS statistical software (V.9; SAS Institute Cary, North Carolina, USA). Two-tailed tests with a significance of $5 \%$ were used throughout. The Pearson $\chi^{2}$ test was used for comparison of different categories, and if any expected frequency of $<5$ was obtained, the Fisher's exact test was used. Survival analysis for breast cancer specific survival (BCSS) was carried out using the Kaplan-Meier method for illustration and log-rank tests for statistical significance. BCSS considered only death due to breast cancer as an event within the first 15 years of follow-up. Death from other causes $(n=11)$ or deaths from breast cancer after 15 years $(n=3)$ were censored. Median follow-up was calculated on patients known to be alive.

The sensitivity and specificity were calculated for a number of Ki67 cut-off values and these are shown in table 1 . The cut-offs $\geq 10$ had the highest Youden's index of $0.346 .^{15}$ Accordingly, Ki67 was divided into 2 categories: $<10$ and $\geq 10$ based on the highest Youden's index. ${ }^{15}$ The outcome factor was breast cancer 
Table 1 Number of deaths, sensitivities and specificities according to a range of cut-off values of Ki67

\begin{tabular}{llllll}
\hline $\begin{array}{l}\text { Ki67 } \\
\text { (\%) }\end{array}$ & $\begin{array}{l}\text { No. died } \\
\text { category }\end{array}$ & $\begin{array}{l}\text { No. in } \\
\text { Sensitivity }\end{array}$ & Specificity & $\begin{array}{l}\text { Youden's } \\
\text { index (J) }\end{array}$ \\
\hline$\geq 0$ & $29(15.0)$ & 193 & 1 & 0 & 0 \\
$\geq 5$ & $28(17.6)$ & 159 & 0.966 & 0.201 & 0.167 \\
$\geq 10$ & $27(22.0)$ & 123 & 0.931 & 0.415 & 0.346 \\
$\geq 15$ & $20(21.3)$ & 94 & 0.690 & 0.549 & 0.238 \\
$\geq 20$ & $16(22.5)$ & 71 & 0.552 & 0.665 & 0.216 \\
$\geq 30$ & $12(25.0)$ & 48 & 0.414 & 0.780 & 0.194 \\
$\geq 40$ & $10(27.8)$ & 36 & 0.345 & 0.841 & 0.186 \\
$\geq 50$ & $8(27.6)$ & 29 & 0.276 & 0.872 & 0.148 \\
\hline \multicolumn{5}{l}{ J=sensitivity+Specificity-1. }
\end{tabular}

survival and the study factors were Ki67 $(<10$ or $\geq 10$; or continuous), age ( $<35$ years, $\geq 35$ years; or continuous), ${ }^{16}$ tumour diameter $(\leq 10 \mathrm{~mm}, 11-15 \mathrm{~mm}, 16-20 \mathrm{~mm}$, or $>20 \mathrm{~mm}$; or continuous), histological grade (1, 2 or 3$)$, ER (positive or negative), LVI (yes or no), mitotic index ( $<10$ or $\geq 10$ ), CK5/6 (positive or negative), HER2 (positive or negative) and contralateral breast cancer (yes or no).

A preliminary univariate analysis was performed with the Kaplan-Meier method, and groups were compared with the log-rank test. Multivariable Cox regression was used to estimate covariate adjusted HR. The selection criterion for predictor inclusion in multivariable analysis was unadjusted or univariate Cox regression $\mathrm{p}$ values $\leq 0.20$.

\section{RESULTS}

The study cohort consisted of 203 patients with primary breast cancer, without lymph node involvement, who underwent breast-conserving surgery followed by radiotherapy. No patient received adjuvant systemic therapy. We conducted analyses comparing the subpopulation and whole initial cohort $(n=421)$ for age, grade, LVI and tumour size. There was no significant difference between means of age. Mean age of subpopulation was 50.4 years $(\mathrm{SD}=10.4)$, compared to 49.3 years $(\mathrm{SD}=10.8)$ of the whole cohort $(p=0.24)$. Similarly, there was no significant difference between means of tumour size (mean $=17.0$ $(\mathrm{SD}=7.5)$ vs mean $=15.9(\mathrm{SD}=7.3), \mathrm{p}=0.07)$. Further, there were no significant differences between the proportions of subpopulation and whole cohort for other characteristics: grade (between $0.6 \%$ (grade 3) and 1.5\% (grade 2, p=0.820)) and LVI $(1 \%, p=0.643)$. The mean age at diagnosis was 50.4 (range, 28-75) years. Cohort characteristics are summarised in table 2. Invasive ductal carcinomas of no special type accounted for $80 \%$ of cases and the tumours were fairly evenly distributed between the three histopathological grades $(33.2 \%$ grade 1 , $34.2 \%$ grade $2,32.6 \%$ grade 3$)$. The majority of cases $(77.2 \%)$ were positive for ER and $69.4 \%$ were positive for PR. HER2 was amplified in only 12 patients (6.2\%) and equivocal in a further 6 patients. After a median follow-up of 183 months (range, 156-277 months), 43 patients (21\%) had died and 32 $(16 \%)$ of these deaths were related to breast cancer.

\section{Ki67 index as a predictor of breast cancer mortality}

Ki67 scores were derived for 193/203 cases. The mean Ki67 index was $22.4 \%$ (range $0.07 \%$ to $85.7 \%$ ).

Increasing Ki67 index was associated with increasing breast cancer mortality across the cohort. The sensitivity and specificity of Ki67 index in 5\% or 10\% increments as a predictor of death
Table 2 Clinicopathological characteristics of 193 women with node-negative breast cancer by Ki67 category

\begin{tabular}{|c|c|c|c|c|c|c|c|}
\hline \multirow{2}{*}{$\begin{array}{l}\text { Patient or tumour } \\
\text { characteristic }\end{array}$} & \multirow[b]{2}{*}{ p Value* } & \multicolumn{2}{|c|}{$\begin{array}{l}\text { Ki67<10 } \\
(n=70)\end{array}$} & \multicolumn{2}{|c|}{$\begin{array}{l}K i 67 \geq 10 \\
(n=123)\end{array}$} & \multicolumn{2}{|c|}{$\begin{array}{l}\text { All } \\
(n=193)\end{array}$} \\
\hline & & $\mathrm{n}$ & $\%$ & $\mathrm{n}$ & $\%$ & $\mathbf{n}$ & $\%$ \\
\hline Age at diagnosis, years & 0.004 & & & & & & \\
\hline$<35$ & & 0 & 0 & 12 & 9.8 & 12 & 6.2 \\
\hline$\geq 35$ & & 70 & 100 & 111 & 90.2 & 181 & 93.8 \\
\hline Tumour size, mm & 0.009 & & & & & & \\
\hline$\leq 10$ & & 20 & 29 & 13 & 10.6 & 33 & 17.2 \\
\hline $11-15$ & & 17 & 24.6 & 47 & 38.2 & 64 & 33.3 \\
\hline $16-20$ & & 18 & 26.1 & 32 & 26 & 50 & 26 \\
\hline$>20$ & & 14 & 20.3 & 31 & 25.2 & 45 & 23.4 \\
\hline Histological grade & $<0.001$ & & & & & & \\
\hline 1 & & 43 & 61.4 & 21 & 17.1 & 64 & 33.2 \\
\hline 2 & & 26 & 37.1 & 40 & 32.5 & 66 & 34.2 \\
\hline 3 & & 1 & 1.4 & 62 & 50.4 & 63 & 32.6 \\
\hline Oestrogen receptor status & $<0.001$ & & & & & & \\
\hline Positive & & 67 & 97.1 & 82 & 67.8 & 149 & 78.4 \\
\hline Negative & & 2 & 2.9 & 39 & 32.2 & 41 & 21.6 \\
\hline LVI & 0.575 & & & & & & \\
\hline No & & 54 & 79.4 & 87 & 71.9 & 141 & 74.6 \\
\hline Yest & & 14 & 20.6 & 34 & 28.1 & 48 & 25.4 \\
\hline Mitotic index & $<0.001$ & & & & & & \\
\hline$<10$ & & 66 & 94.3 & 60 & 48.8 & 126 & 65.3 \\
\hline$\geq 10$ & & 4 & 5.7 & 63 & 51.2 & 67 & 34.7 \\
\hline CK5/6 & 0.001 & & & & & & \\
\hline Negative & & 58 & 84.1 & 72 & 60 & 130 & 68.8 \\
\hline Positive & & 11 & 15.9 & 48 & 40 & 59 & 31.2 \\
\hline HER2 & 0.004 & & & & & & \\
\hline Negative & & 70 & 100 & 108 & 90.0 & 178 & 93.7 \\
\hline Positive & & 0 & 0 & 12 & 10.0 & 12 & 6.3 \\
\hline Contralateral & 0.749 & & & & & & \\
\hline Yes & & 3 & 4.3 & 8 & 6.5 & 11 & 5.7 \\
\hline No & & 67 & 95.7 & 115 & 93.5 & 182 & 94.3 \\
\hline
\end{tabular}

${ }^{*} p$ Values are for comparison of categories of each variable by Ki67 using the Pearson $\chi^{2}$ test or Fisher's exact test; 193 of 203 cases were evaluable for Ki67. tIncludes three indeterminate cases.

CK, cytokeratin; HER2, human epidermal growth factor 2; LVI, lymphovascular invasion.

from breast cancer is seen in table 1. The highest Youden's index for Ki67 index is seen at $\geq 10 \%$, where the sensitivity is 0.931 . The specificity is seen to increase as the Ki67 count increases with the highest specificity seen when the count is $\geq 50 \%$, where the specificity is 0.872 . A cut-off of $\geq 10 \%$ had the highest Youden's index of 0.346, accordingly, the Ki67 index was used to specify two subgroups: $<10 \%$ (Ki67-low, $\mathrm{n}=70$ ) and $\geq 10 \%$ (Ki67-high, $\mathrm{n}=123$ ).

The striking difference in mortality between patients in the Ki67-low and Ki67-high subgroups is illustrated in figure 1. Only 2 of the 70 patients (3\%) in the Ki67-low group died of breast cancer compared with 27 of 123 patients $(22 \%)$ in the Ki67-high group $(\mathrm{p}<0.001)$. The overall 15 -year actuarial survival for the Ki67-low subgroup was 97\% compared with 78\% for Ki67-high ( $p=0.0003$; figure 1). It was further noted that the two breast cancer deaths that occurred in the Ki67-low subgroup were both more than 10 years after diagnosis. In contrast, in the Ki67-high subgroup, 23/27 deaths (85\%) occurred within 10 years after diagnosis, with 15 of these occurring within the first 5 years. 


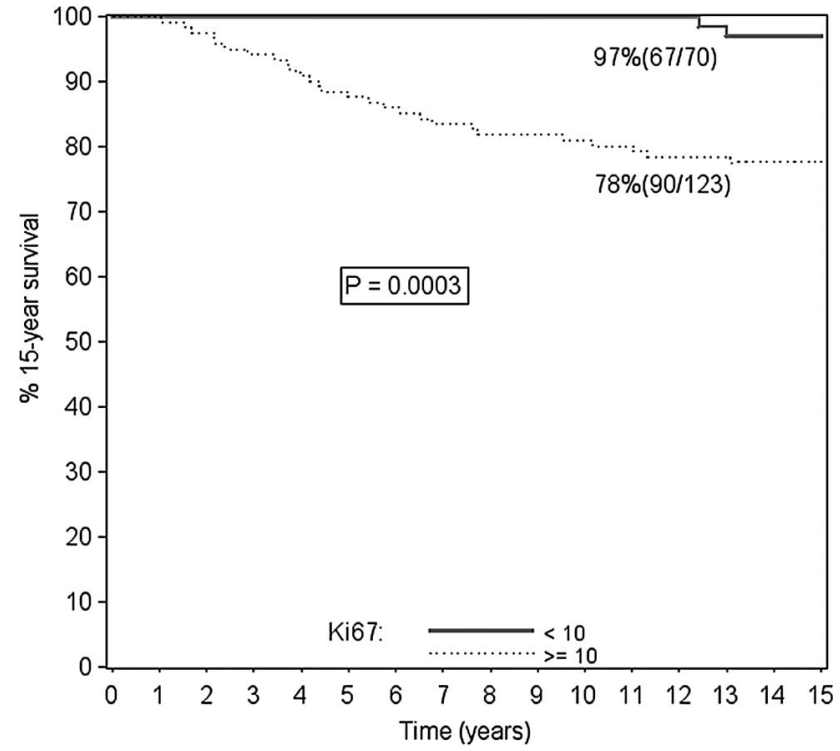

Figure 1 Overall 15-year survival for low and high Ki67 tumours. Numbers in parentheses indicate total number and number at risk.

\section{Clinicopathological features of Ki67-low and Ki67-high subgroups}

The clinicopathological features of Ki67-low and Ki67-high subgroups were distinct (table 2). All of the cases diagnosed in individuals aged $<35$ years belonged to the Ki67 high subgroup $(\mathrm{p}=0.004)$. The Ki67 high subgroup was also associated with larger tumour size $(\mathrm{p}=0.009)$, higher grade $(\mathrm{p}<0.001)$ and greater proportion of ER-negative tumours $(\mathrm{p}<0.001)$. A mitotic index of $\geq 10$ was seen in $51.2 \%$ of Ki67-high tumours compared with only $5.7 \%$ of Ki67-low tumours $(p=<0.001)$. Expression of CK5/6 was seen more frequently in the Ki67-high subgroup with $40.0 \%$ of this group positive compared with $15.9 \%$ of the Ki67-low subgroup $(\mathrm{p}=0.001)$. All cases of HER2-positive disease were in the Ki67-high group $(p=0.004)$. Patients with a high Ki67 index showed similar rates of mortality in the ER-positive and ER-negative groups, $22.0 \%$ and $23.1 \%(\mathrm{p}=0.89)$, respectively (table 3$)$.

\section{Comparison of Ki67 index and other clinicopathological features as prognostic factors}

Univariate analysis showed that in this lymph node-negative cohort, the predictors for BCSS were tumour size, Ki67, LVI, age and histological grade 3(table 4). Histological grade 2, ER, CK5/6, HER2, contralateral breast cancer and mitotic index were not found to be statistically significant predictors of BCSS.

By multivariable analysis with simultaneous adjustment for age, tumour size, grade, ER status, mitotic index $>10$ and CK5/

Table 3 Breast cancer deaths by oestrogen receptor status and Ki67 category

\begin{tabular}{|c|c|c|c|c|c|}
\hline \multirow[b]{2}{*}{ Ki67 group } & \multicolumn{2}{|c|}{$\begin{array}{l}\text { Oestrogen receptor } \\
\text { positive }\end{array}$} & \multicolumn{2}{|c|}{$\begin{array}{l}\text { Oestrogen receptor } \\
\text { negative }\end{array}$} & \multirow[b]{2}{*}{ p Value } \\
\hline & $\mathrm{n}$ & $\begin{array}{l}\text { Breast cancer } \\
\text { deaths }(\%)\end{array}$ & $\mathbf{n}$ & $\begin{array}{l}\text { Breast cancer } \\
\text { deaths }(\%)\end{array}$ & \\
\hline Ki67-high & 82 & $18(22.0)$ & 39 & $9(23.1)$ & 0.89 \\
\hline Ki67-low & 67 & $2(3.0)$ & 2 & $0(0)$ & 1 \\
\hline p Value & \multicolumn{2}{|c|}{0.001} & 1 & & \\
\hline
\end{tabular}

6 positivity, only Ki67 index $(\mathrm{p}=0.005)$ and LVI $(\mathrm{p}=0.003)$ remained as independent prognostic factors for BCSS (table 4).

\section{Defining surrogate molecular subtypes using Ki67}

A study by Cheang $e t ~ a l^{17}$ that specifically compared a gene expression based intrinsic subtype classification of breast cancers with pathological features, reported that a Ki67 score $\geq 14 \%$ distinguished Luminal B from Luminal A, ER-positive HER2-negative tumours. This approach was subsequently supported by the St Gallen 2011 Expert Panel ${ }^{18}$ as an alternative to molecular subtyping. In order to examine the utility of Ki67 in the context of this classifier, cases were assigned into Luminal A (ER-positive, HER2-negative, Ki67 $\leq 14 \%$ ), Luminal B (ER-positive, HER2-negative, Ki67 >14\%) and triple negative (ER/PR-negative and HER2-negative, any Ki67) intrinsic subtype categories.

The 15 -year breast cancer specific survival of women with luminal A was $91.7 \%$, compared with $79.4 \%$ for luminal B $(\mathrm{p}=0.027)$ and $75.8 \%$ for triple negative $(\mathrm{p}=0.010)$, respectively (table 5 , figure 2). A statistically significant difference in BCSS was seen between the four subgroups $(p=0.009$, figure 2). Histological grading, by contrast was not a significant predictor of survival. Histological grading information was available for 201 patients in the cohort, with 67 grade 1,70 grade 2 and 64 grade 3 tumours. There were 4 deaths (6.0\%) from breast cancer in the grade 1 group, $6(8.6 \%)$ in the grade 2 group and $11(17.2 \%)$ in the grade 3 group. The survival differences between the two groups did not reach statistical significance $(p=0.106)$.

\section{DISCUSSION}

The impact of cancer cell proliferation on breast cancer prognosis has been long acknowledged, and in traditional histopathological grading, this assessment is captured by inclusion of a mitotic count score as one of the three components simultaneously assessed. Recently, a more detailed assessment of breast cancer biology has been promoted by molecular profiling studies, which define breast cancer subtypes, as well as individual molecular features and pathways that correlate with prognosis. Cellular proliferation has emerged as a key discriminative feature of malignant phenotype from this work. A labelling index based on immunohistochemical staining of the cell cycle antigen Ki67, is a widely accessible method to make this assessment.

In this study, Ki67 was found to be an independent prognostic factor for 15 year BCSS, in a series of patients with nodenegative breast cancer previously untreated with adjuvant systemic therapy. All patients were treated with the standard regime of breast conserving surgery with axillary dissection and radiation therapy. Univariate analysis of Ki67 index along with other routinely assessed clinicopathological variables found LVI, Ki67 index, tumour size, age and histological grade 3 to be statistically significant prognostic factors for BCSS. On multivariable analysis, only Ki67 index and LVI persisted as significant and independent predictors of outcome. Importantly, mitotic rate was not found to be of prognostic value in this cohort, which suggests that Ki67 is a more powerful and sensitive means of assessing tumour proliferation.

Currently there are a number of commercial gene expression based assays available to assess intrinsic breast cancer subtype and gene prognostic scores. ${ }^{4}{ }^{19}$ Proliferation is a key discriminative component in these assays and it has been demonstrated that similar information can be derived from more routine pathological assessment when a Ki67 score is included. For 
Table 4 Univariate and multivariate analysis of Ki67 and other clinicopathological characteristics at 15 years of follow-up

\begin{tabular}{|c|c|c|c|c|c|c|c|c|c|}
\hline \multirow[b]{2}{*}{ Feature } & \multirow[b]{2}{*}{ Rate (\%) } & \multicolumn{2}{|l|}{ Survival } & \multicolumn{3}{|c|}{ Univariate analysis } & \multicolumn{3}{|c|}{ Multivariate analysis } \\
\hline & & $95 \% \mathrm{Cl}$ & p Value* & $\mathbf{R R}$ & $95 \% \mathrm{Cl}$ & $\mathrm{p}$ Value & $\mathbf{R R}$ & $95 \% \mathrm{Cl}$ & $p$ Value \\
\hline \multicolumn{10}{|l|}{ Ki67 } \\
\hline$<10$ & 97.1 & 93.2 to 100 & $<0.001$ & 1 & & & 1 & & \\
\hline$\geq 10$ & 77.6 & 70.2 to 85.0 & & 8.88 & 2.11 to 37.35 & 0.003 & 7.96 & 1.69 to 37.48 & 0.009 \\
\hline \multicolumn{10}{|l|}{ Aget } \\
\hline$<35$ & 66.7 & 40.0 to 93.4 & 0.051 & 2.72 & 0.95 to 7.82 & 0.063 & 1.59 & 0.52 to 4.84 & 0.414 \\
\hline$\geq 35$ & 86.1 & 81.2 to 91.0 & & 1 & & & 1 & & \\
\hline \multicolumn{10}{|l|}{ Tumour size } \\
\hline$\leq 10$ & 94.1 & 86.3 to 100 & & 1 & & & 1 & & \\
\hline $11-15$ & 89.3 & 81.9 to 96.7 & 0.444 & 1.78 & 0.37 to 8.58 & 0.471 & 0.79 & 0.16 to 4.03 & 0.778 \\
\hline $16-20$ & 82.8 & 72.6 to 93.0 & 0.136 & 3.04 & 0.66 to 14.09 & 0.155 & 1.39 & 0.28 to 6.96 & 0.689 \\
\hline$>20$ & 73.7 & 61.0 to 86.4 & 0.02 & 4.35 & 096 to 19.63 & 0.056 & 1.73 & 0.32 to 9.30 & 0.521 \\
\hline \multicolumn{10}{|c|}{ Histological grade } \\
\hline 1 & 89.2 & 81.8 to 96.6 & & 1 & & & 1 & & \\
\hline 2 & 87.1 & 79.3 to 94.9 & 0.659 & 1.5 & 0.53 to 4.20 & 0.446 & 0.81 & 0.27 to 2.39 & 0.699 \\
\hline 3 & 77.8 & 67.6 to 88.0 & 0.055 & 2.72 & 1.05 to 7.08 & 0.04 & 1.11 & 0.27 to 4.52 & 0.887 \\
\hline \multicolumn{10}{|c|}{ Oestrogen receptor status } \\
\hline Positive & 86.6 & 81.1 to 92.1 & 0.125 & 0.54 & 0.25 to 1.19 & 0.126 & 0.96 & 0.33 to 2.81 & 0.934 \\
\hline Negative & 78.6 & 66.3 to 90.9 & & 1 & & & 1 & & \\
\hline \multicolumn{10}{|l|}{ LVI } \\
\hline No & 89.8 & 84.9 to 94.7 & 0.001 & 1 & & & 1 & & \\
\hline Yes‡ & 70.5 & 57.6 to 83.4 & & 3.17 & 1.51 to 6.66 & 0.002 & 2.77 & 1.26 to 6.11 & 0.012 \\
\hline \multicolumn{10}{|c|}{ Mitotic index§ } \\
\hline$<10$ & 87.8 & 82.1 to 93.5 & 0.076 & 1 & & & 1 & & \\
\hline$\geq 10$ & 79.1 & 69.3 to 88.9 & & 1.95 & 0.94 to 4.04 & 0.072 & 0.6 & 0.18 to 1.98 & 0.4 \\
\hline \multicolumn{10}{|l|}{ CK5/6 } \\
\hline Negative & 87.2 & 81.5 to 92.9 & 0.186 & 1 & & & 1 & & \\
\hline Positive & 79.7 & 69.5 to 89.9 & & 1.62 & 0.77 to 3.38 & 0.203 & 1.23 & 0.55 to 2.76 & 0.622 \\
\hline \multicolumn{10}{|l|}{ HER2 } \\
\hline Negative & 84.8 & 79.5 to 90.1 & 0.583 & 1 & & 0.588 & & & \\
\hline Positive & 91.7 & 76.0 to 100 & & 0.58 & 0.08 to 4.24 & & & & \\
\hline \multicolumn{10}{|c|}{ Contralateral } \\
\hline Yes & 72.7 & 46.4 to 99.0 & 0.198 & 2.13 & 0.65 to 7.05 & 0.214 & & & \\
\hline No & 85.6 & 80.5 to 90.7 & & 1 & & & & & \\
\hline
\end{tabular}

example, a study by Cheang $e t a l^{17}$ that specifically compared a gene expression based intrinsic subtype classification of breast cancers with pathological features, reported that a Ki67 score $\geq 14 \%$ distinguished Luminal B from Luminal A, ER-positive HER2-negative tumours. Similarly, Cuzick et al demonstrated ${ }^{20}$ that an algorithm based on semiquantitative scores for ER and PR expression, HER2 status a Ki67 score derived from immmunohistochemical staining ('IHC-4' score) showed similar prognostic performance to the 21-gene Recurrence Score for ER-positive breast cancer. Taken together these and the current studies illustrate a key role for Ki67 score in current approaches to prognostic assessment of ER-positive breast cancer. Moreover, the value of Ki67 as an individual biomarker for breast cancer prognostication has been reported in a large number of studies and in several meta-analyses. ${ }^{21-24}$ This validation of Ki67 as a prognostic marker remains robust despite marked variation in study methods, design and cut-off values.

In the current study, a cut off of $10 \%$ for Ki67 positivity was found to be prognostically discriminative across the entire cohort. These findings are consistent with those of a recent larger study of 1550 cases by Aleskandarany et al. ${ }^{11}$ In this study a $10 \%$ cut off for Ki67 stratified grade 1 and grade 2 breast cancers into statistically significant prognostic groups. In addition, the study by these authors found that Ki67 index was the strongest predictor of overall and metastasis free survival in univariate and multivariate analysis in grade 2 tumours. Of note about half of their 506 grade 2 tumours, had a Ki67 level of under $10 \%$ ('grade 2a') and half $10 \%$ or more ('grade 2 b'). Grade $2 \mathrm{~b}$ tumours had a $76 \%$ 10-year BCSS rate compared to $92 \%$ in the grade 2 a cases.

In the current study all the patients in the Ki67-low group had a good prognosis, with few deaths from breast cancer in the cohort at 15 years; those deaths that did occur were seen after 10 years. This is indicative of the long clinical course of these tumours and carries significant implications for patient follow-up protocols. By contrast, Ki67-high tumours showed a steady rate of death from cancer in the first 5-10 years, plateauing thereafter. 
Table 5 Sublassification into molecular subtypes and number of deaths in each category

\begin{tabular}{lrl}
\hline Molecular subtype & No. & No. of deaths (\%) \\
\hline Luminal A & 86 & $3(96.5 \%)$ \\
Luminal B & 59 & $8(86.4 \%)$ \\
HER2 positive & 11 & 0 \\
Triple negative & 34 & $8(76.5 \%)$ \\
Total & 190 & $19(90 \%)$ \\
\hline HER2, human epidermal growth factor 2. &
\end{tabular}

None of the women in this study received any form of adjuvant therapy; this presented an opportunity to evaluate the true biological nature of breast carcinoma without the confounding effects of systemic therapy. The marked survival differences in grade 1 tumours in our cohort are partially explained by the lack of hormonal treatment in these women, emphasising the effect of treatment on prognosis. In our study, patients with a high Ki67 index showed similar rates of mortality in the ER-positive and ER-negative groups, $22.0 \%$ and $23.1 \%$ $(p=0.89)$, respectively (table 3$)$.

Ki67 as an individual biomarker has been confirmed in this and other studies as well as several meta-analyses. In this study we have also demonstrated the utility of Ki67 in classifying breast cancers into surrogate molecular subtypes; this approach was subsequently supported by the St Gallen 2011 Expert Panel as an alternative to molecular subtyping. ${ }^{18} \mathrm{~A}$ statistically significant difference in BCSS is seen in the four subgroups defined using Ki67 and receptor status $(\mathrm{p}=0.009$, figure 2). Histological grading in this study, by contrast was not a significant predictor of survival $(p=0.106)$. Some authorities strongly advocate the inclusion of Ki67 along with ER, PR and HER2 as useful for defining breast cancer subtypes with high Ki67 levels warranting consideration of chemotherapy. ${ }^{25}$ Clearly, pure morphological evaluation of breast cancers is limited by its subjectivity and recently there have been several studies attempting to replicate molecular classifications of breast cancer using immunohistochemistry. $^{26} \mathrm{Ki} 67$ is a good candidate in this respect, and as shown, appears to be more robust and less subjective than mitotic index. This prognostic classification of breast cancer has the advantage of providing information relating to outcome as well as serving as a guide for possible assignment into various therapeutic pathways.

A potential barrier to the clinical utility of Ki67 is the lack of consensus regarding the optimal percentage positive cut-off value to use as well as the methodology that should be adopted. Estimates of Ki67 positivity are likely to be poorly reproducible, and in this study we used an individual cell counting method using scanned images, facilitated by the use of software with a manual tag function to assist with counting. Multiple TMA cores from each tumour were examined to minimise the effects of tumour heterogeneity. In practice however, Ki67 is likely to be used in whole tumour sections. In the study by Aleskandarany et al, 'hot spots' were evaluated to account for areas which are likely to drive the biological potential of the tumour, analogous to the approach taken in Scarff-BloomRichardson grading where the tumour periphery is scanned for the most mitotically active area to assess the mitotic score. An alternative approach aims to derive a representative score by selecting fields with a range of staining patterns across the section. Clearly, additional work in this area to standardise approaches is required. ${ }^{11}$
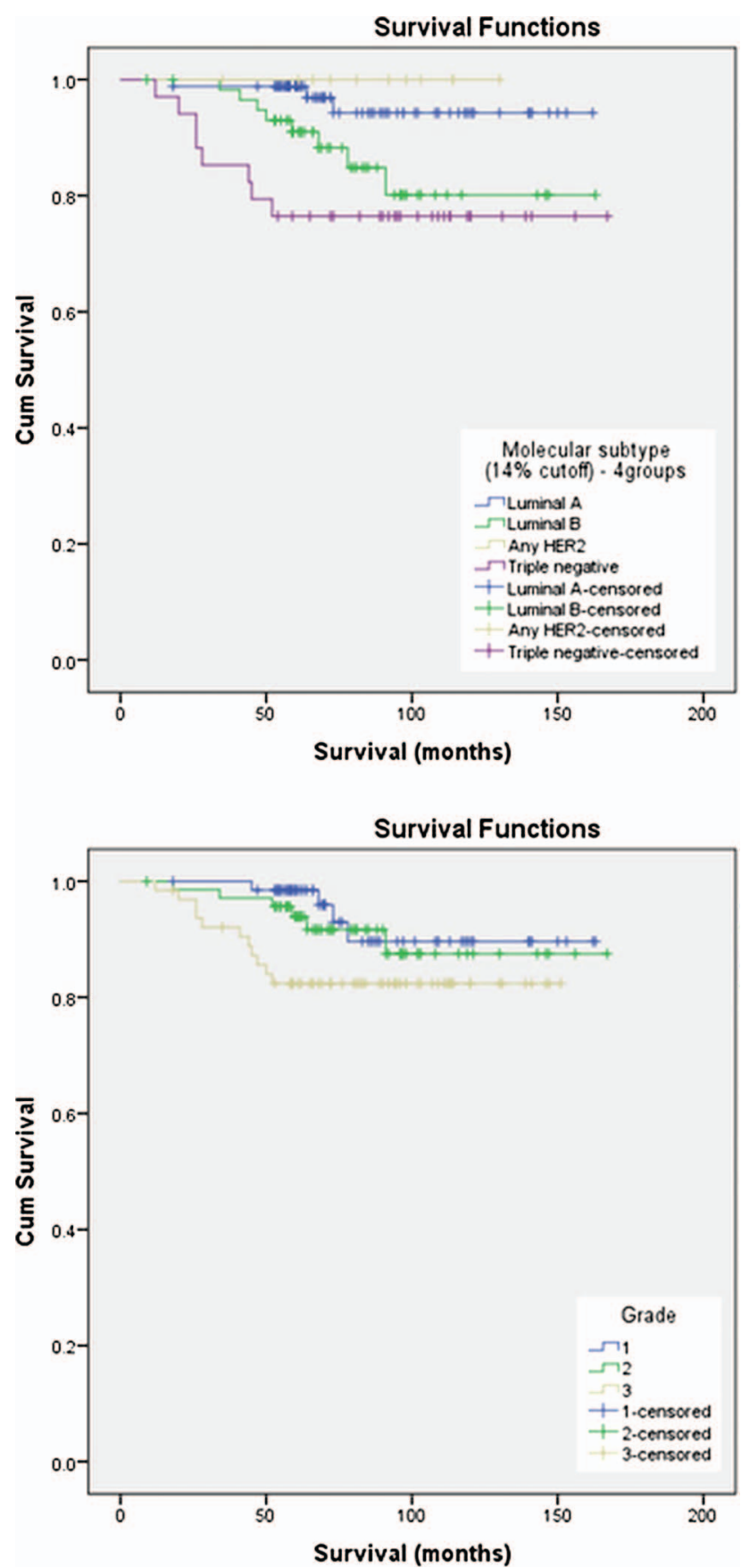

Figure 2 Breast cancer specific survival by surrogate molecular subtype and grade.

Limitations of the current study include the small sample numbers and the use of TMAs in the assessment, which is unlikely to reflect clinical practice. The study demonstrates however, the utility of an individual cell counting method in objective Ki67 assessment. It also demonstrates the potential ways in which Ki67 can be used as a prognostic marker. This study is best regarded as an exploratory study. Additional studies are required to validate and standardise methodology for Ki67 in routine practice.

In conclusion, this study has confirmed the prognostic value of Ki67 assessment in a cohort of node-negative, systemic treatment-naïve patients with breast cancer with 15 years of follow-up. Analysis of multiple clinicopathological variables revealed that Ki67 index was the most powerful and independent predictor of survival. This cost effective, widely available 
method provides prognostic information beyond traditionally assessed clinicopathological variables and could enhance treatment planning and prognostication, especially relevant to the challenging area of early breast cancer.

\section{Take home messages}

- A labelling index based on immunohistochemical staining of the cell cycle antigen Ki67, is a widely accessible method to assess cellular proliferation and is a powerful predictor of survival.

- Ki67 assessment provides prognostic information beyond traditionally assessed clinicopathological variables.

- Ki67 assessment may have a key role in the prognostic assessment of oestrogen receptor positive breast cancer and provide additional information for therapeutic decisions.

- Additional studies are required to validate and standardise methodology for Ki67 in routine practice.

\section{Author affiliations}

Westmead Breast Cancer Institute, Westmead Hospital, Westmead, New South Wales, Australia

${ }^{2}$ Department of Tissue Pathology and Diagnostic Oncology, Institute of Clinical Pathology and Medical Research, Westmead Hospital, New South Wales, Australia ${ }^{3}$ Sydney Medical School, Westmead, University of Sydney, Sydney, New South Wales, Australia

${ }^{4}$ Translational Oncology, Western Sydney Local Health District, Sydney, New South Wales, Australia

${ }^{5}$ Westmead Institute for Cancer Research, Sydney, New South Wales, Australia

${ }^{6}$ Westmead Millennium Institute, NSW Australia, Sydney, New South Wales, Australia

${ }^{7}$ University of New South Wales, Sydney, New South Wales, Australia

${ }^{8}$ Department of Healthscope Pathology, Bella Vista, New South Wales, Australia

${ }^{9}$ Department of Anatomical Pathology, Prince of Wales Hospital, Randwick, New South Wales, Australia

${ }^{10}$ Macquarie University Cancer Institute, North Ryde, New South Wales, Australia

Contributors NP, JB were involved in the concept and development of this study. $N P, J B$ and $K B$ were responsible for initial data collection. NP, AMB, ELS were responsible for pathological analysis. KB and UWJ were responsible for statistical analysis. The first author was responsible for the first draft of the paper and remaining authors for further contribution and review.

Competing interests NP and AMB declare receipt of speaker honoraria and related travel support from Roche Pty Ltd. AMB is a member of the Hoffmann La Roche HER2 Testing Advisory Board. PJP and RLB gratefully acknowledge support from the Cancer Institute NSW (CINSW). RLB is a CINSW Fellow.

Ethics approval Sydney West Local Health District Human Research Ethics Committee.

Provenance and peer review Not commissioned; externally peer reviewed.

Open Access This is an Open Access article distributed in accordance with the Creative Commons Attribution Non Commercial (CC BY-NC 4.0) license, which permits others to distribute, remix, adapt, build upon this work non-commercially, and license their derivative works on different terms, provided the original work is properly cited and the use is non-commercial. See: http://creativecommons.org/ licenses/by-nc/4.0/

\section{REFERENCES}

1 Perou CM, Sorlie T, Eisen MB, et al. Molecular portraits of human breast tumours. Nature 2000;406:747-52.
2 Sorlie T, Perou CM, Tibshirani R, et al. Gene expression patterns of breast carcinomas distinguish tumour subclasses with clinical implications. Proc Natl Acad Sci 2001;98:10869-74.

3 Prat A, Ellis MJ, Perou CM. Practical implications of gene-expression-based assays for breast oncologists. Nat Rev Clin Oncol 2011;9:48-57.

4 Paik S, Shak S, Tang G, et al. A multigene assay to predict recurrence of tamoxifen-treated, node-negative breast cancer. N Engl J Med 2004;351:2817-26.

5 Berry DA, Cirrincione C, Henderson IC, et al. Estrogen-receptor status and outcomes of modern chemotherapy for patients with node-positive breast cancer. JAMA 2006:295:1658-67.

6 Boyages J, Taylor R, Chua B, et al. A risk index for early node-negative breast cancer. Br J Surg 2006;93:564-71.

7 Fisher B, Slack NH. Number of lymph nodes examined and the prognosis of breast carcinoma. Surg Gynecol Obstet 1970;131:79-88.

8 Tavassoli PDFA. World Health Organization: tumours of the breast and female genital organs. Oxford, Oxfordshire: Oxford University Press, 2003.

9 Elston CW, Ellis IO. Pathological prognostic factors in breast cancer. I. The value of histological grade in breast cancer: experience from a large study with long-term follow-up. Histopathology 1991;19:403-10.

10 Bloom HJ, Richardson WW. Histological grading and prognosis in breast cancer; a study of 1409 cases of which 359 have been followed for 15 years. Br J Cancer 1957;11:359-77.

11 Aleskandarany MA, Rakha EA, Macmillan RD, et al. MIB1/Ki-67 labelling index can classify grade 2 breast cancer into two clinically distinct subgroups. Breast Cancer Res Treat 2011:127:591-9.

12 Rakha EA, El-Sayed ME, Green AR, et al. Breast carcinoma with basal differentiation: a proposal for pathology definition based on basal cytokeratin expression. Histopathology 2007;50:434-8.

13 Wolff AC, Hammond ME, Schwartz JN, et al. American Society of Clinical Oncologyl College of American Pathologists guideline recommendations for human epidermal growth factor receptor 2 testing in breast cancer. Arch Pathol Lab Med 2007;131:18-43.

14 Penault-Llorca F, Bilous M, Dowsett M, et al. Emerging technologies for assessing HER2 amplification. Am J Clin Pathol 2009:132:539-48.

15 Bewick V, Cheek L, Ball J. Statistics review 13: receiver operating characteristic curves. Crit Care 2004:8:508-12

16 Jayasinghe UW, Taylor R, Boyages J. Is age at diagnosis an independent prognostic factor for survival following breast cancer? ANZJ Surg 2005;75:762-7.

17 Cheang MCU, Chia SK, Voduc D, et al. Ki67 Index, HER2 Status, and Prognosis of Patients With Luminal B Breast Cancer. J Natl Cancer Inst 2009;101:736-50.

18 Goldhirsch A, Wood WC, Coates AS, et al. Strategies for subtypes--dealing with the diversity of breast cancer: highlights of the St. Gallen International Expert Consensus on the Primary Therapy of Early Breast Cancer 2011. Ann Oncol 2011;22:1736-47

19 Parker JS, Mullins M, Cheang MC, et al. Supervised risk predictor of breast cancer based on intrinsic subtypes. J Clin Oncol 2009;27:1160-7.

20 Cuzick J, Dowsett M, Pineda S, et al. Prognostic value of a combined estrogen receptor, progesterone receptor, Ki-67, and human epidermal growth factor receptor 2 immunohistochemical score and comparison with the Genomic Health recurrence score in early breast cancer. J Clin Oncol 2011;29:4273-8.

21 Luporsi E, Andre F, Spyratos F, et al. Ki-67: level of evidence and methodological considerations for its role in the clinical management of breast cancer: analytical and critical review. Breast Cancer Res Treat 2012;132:895-915

22 Urruticoechea A, Smith IE, Dowsett M. Proliferation marker Ki-67 in early breas cancer. J Clin Oncol 2005;23:7212-20.

23 Stuart-Harris R, Caldas C, Pinder SE, et al. Proliferation markers and survival in early breast cancer: a systematic review and meta-analysis of 85 studies in 32,825 patients. Breast 2008;17:323-34.

24 de Azambuja E, Cardoso F, de Castro G Jr, et al. Ki-67 as prognostic marker in early breast cancer: a meta-analysis of published studies involving 12,155 patients. Br J Cancer 2007:96:1504-13.

25 Dowsett M, Nielsen TO, ÄôHern R, et al. Assessment of Ki67 in Breast Cancer: Recommendations from the International Ki67 in Breast Cancer Working Group. J Natl Cancer Inst 2011;103:1656-64.

26 Blows FM, Driver KE, Schmidt MK, et al. Subtyping of breast cancer by immunohistochemistry to investigate a relationship between subtype and short and long term survival: a collaborative analysis of data for 10,159 cases from 12 studies. PLoS Med 2010;7:e1000279. 